\title{
Multiple Solutions for Boundary Value Problems of $p$-Laplacian Difference Equations Containing Both Advance and Retardation
}

\author{
Zhenguo Wang ${ }^{1,2}$ and Zhan Zhou ${ }^{1}{ }^{1}$ \\ ${ }^{1}$ School of Mathematics and Information Science, Guangzhou University, Guangzhou 510006, China \\ ${ }^{2}$ Department of Mathematics, Lüliang University, Lüliang 033000, China
}

Correspondence should be addressed to Zhan Zhou; zzhou0321@hotmail.com

Received 5 May 2020; Accepted 1 June 2020; Published 10 August 2020

Guest Editor: Chuangxia Huang

Copyright (c) 2020 Zhenguo Wang and Zhan Zhou. This is an open access article distributed under the Creative Commons Attribution License, which permits unrestricted use, distribution, and reproduction in any medium, provided the original work is properly cited.

This paper concerns the existence of solutions for the Dirichlet boundary value problems of $p$-Laplacian difference equations containing both advance and retardation depending on a parameter $\lambda$. Under some suitable assumptions, infinitely many solutions are obtained when $\lambda$ lies in a given open interval. The approach is based on the critical point theory.

\section{Introduction}

Let $\mathbb{Z}$ and $\mathbb{R}$ be the sets of integers and real numbers, respectively. For $a, b \in \mathbb{Z}, \mathbb{Z}(a, b)$ denotes the discrete interval $\{a, a+1, \ldots, b\}$ if $a \leq b$.

Assume that $N$ is a positive integer. In this paper, we consider the following boundary value problem of difference equation containing both advance and retardation:

$$
\left\{\begin{array}{l}
-\Delta\left(\phi_{p}\left(\Delta u_{k-1}\right)\right)+2 q_{k} \phi_{p}\left(u_{k}\right)=\lambda f\left(k, u_{k+1}, u_{k}, u_{k-1}\right), \\
\quad k \in \mathbb{Z}(1, N), \\
u_{0}=u_{N+1}=0
\end{array}\right.
$$

where $\lambda$ is a positive real parameter, the forward difference operator is defined as $\Delta u_{k}=u_{k+1}-u_{k}$. For every $k \in \mathbb{Z}(1, N), \quad q_{k} \geq 0, \quad f(k, \cdot): \mathbb{R}^{3} \longrightarrow \mathbb{R}$ is a continuous function, $\phi_{p}(s)$ is the $p$-Laplacian operator, that is, $\phi_{p}(s)=|s|^{p^{-2}} s$, and $1<p<+\infty$.

As it is to know, difference equations have been widely used in various research fields such as computer science, economics, network, and control system. Relevant examples and mathematical models can be found in [1-3]. By means of critical point theory, many researchers devote themselves to the study of difference equations and achieve many excellent results, for example, the results on boundary value problems [4-16], periodic solutions [17-22], and homoclinic solutions [23-31] had been obtained.

Difference equations containing both advance and retardation have many applications in physical and biological phenomena [32]. Yu et al. [23] obtained the existence of nontrivial homoclinic orbits for the following second-order difference equation containing both advance and retardation:

$$
L u_{k}-\omega u_{k}=f\left(k, u_{k+T}, u_{k}, u_{k-T}\right), \quad k \in \mathbb{Z} .
$$

The operator $L$ is a second-order difference operator given by

$$
L u_{k}=a(k) u_{k+1}+a(k-1) u_{k-1}+b(k) u_{k},
$$

where $a(k)$ and $b(k)$ are real valued for each $k \in \mathbb{Z}$ and $\omega \in \mathbb{R}$.

Mei and Zhou [21] considered the existence of the periodic and subharmonic solutions of a $2 n$th order $p$-Laplacian difference equation containing both advances and retardations:

$$
\begin{gathered}
\Delta^{n}\left(r_{k-n} \varphi_{p}\left(\Delta^{n} u_{k-n}\right)\right)=(-1)^{n} f\left(k, u_{k+\tau}, \ldots, u_{k+1}, u_{k}, u_{k-1},\right. \\
\left.\ldots, u_{k-\tau}\right), \quad k \in \mathbb{Z} .
\end{gathered}
$$


There are more about the results of the difference equations containing both advance and retardation which can be seen in $[13,19,22,24]$.

Here, we are interested in using critical point theory to investigate infinitely many solutions for (1) containing both advance and retardation.

In fact, there are some papers which studied the existence of infinitely many solutions for the boundary value problems of difference equations. Bonanno and Candito [33] in 2009 proved the existence of infinitely many solutions of the following discrete boundary value problem:

$$
\left\{\begin{array}{l}
-\Delta\left(\phi_{p}\left(\Delta u_{k-1}\right)\right)+q_{k} \phi_{p}\left(u_{k}\right)=\lambda f\left(k, u_{k}\right), \quad k \in \mathbb{Z}(1, N), \\
u_{0}=u_{N+1}=0 .
\end{array}\right.
$$

Recently, Zhou and Ling [10] studied the existence of infinitely many positive solutions for the following boundary value problem of the second order nonlinear difference equation with $\phi_{c}$-Laplacian:

$$
\left\{\begin{array}{l}
-\Delta\left(\phi_{c}\left(\Delta u_{k-1}\right)\right)=\lambda f\left(k, u_{k}\right), \quad k \in \mathbb{Z}(1, N), \\
u_{0}=u_{N+1}=0 .
\end{array}\right.
$$

However, to the best of our knowledge, no similar results are obtained for problem (1) containing both advance and retardation. The main difficulty is caused by the advance and retardation. In this paper, we obtain some sufficient conditions to guarantee the existence of infinitely many solutions of (1). In fact, under some assumptions, we prove the existence of infinitely many solutions of equation (1) for each $\lambda \in\left((2+2 Q) /(p B), 2 /\left(p(N+1)^{p-1} A\right)\right)$ in Theorem 1 . Moreover, Theorem 2 guarantees the existence of infinity positive solutions for (1) by applying a strong maximum principle. Finally, we show that (1) possesses a sequence of distinct solutions which converges to zero for each $\lambda \in((2+$ $\left.2 Q) / p B^{*}, 2^{p-1}(1+q) / p(N+1)^{p-1} A^{*}\right)$ in Theorem 3 .

This paper is organized as follows. In Section 2, some definitions and preliminaries on difference equations are collected. In Section 3, our main results are established. Finally, two examples are given to illustrate our main results.

\section{Preliminaries}

Let $E$ be a reflexive real Banach space and $I_{\lambda}: E \longrightarrow \mathbb{R}$ be a function satisfying the following structure hypothesis: $\left(H_{1}\right)$ Assume that $\lambda$ is a real positive parameter. $I_{\lambda}:=\Phi(u)-\lambda \Psi(u), \forall u \in E$, where $\Phi, \Psi \in C^{1}(E, \mathbb{R}), \Phi$ is coercive, that is, $\lim _{\|u\| \rightarrow \infty} \Phi(u)=+\infty$.

Provided that $\inf _{E} \Phi<r$, write

$$
\begin{aligned}
\varphi(r) & =\inf _{v \in \Phi^{-1}(-\infty, r)} \frac{\sup _{v \in \Phi^{-1}(-\infty, r)} \Psi(v)-\Psi(u)}{r-\Phi(u)}, \\
\gamma & =\liminf _{r \longrightarrow+\infty} \varphi(r), \\
\delta & =\liminf _{r \longrightarrow\left(\inf _{E} \Phi\right)^{+}} \varphi(r) .
\end{aligned}
$$

Obviously, $\gamma \geq 0$ and $\delta \geq 0$. When $\gamma=0$ or $\delta=0$, we agree to $\mathrm{read} 1 / \gamma$ or $1 / \delta$ as $+\infty$.

Lemma 1 (see [34]). Assume that condition $\left(H_{1}\right)$ holds, and one has

(a) For every $r>\inf \Phi$ and every $\lambda \in(0,1 / \varphi(r))$, the restriction of ${ }^{E}$ unctional $I_{\lambda}=\Phi(u)-\lambda \Psi(u)$ to $u \in \Phi^{-1}(-\infty, r)$ admits a global minimum, which is a critical point (local minimum) of $I_{\lambda}$ in $E$.

(b) If $\gamma<+\infty$, then for each $\lambda \in(0,1 / \gamma)$, the following alternative holds:

$\left(b_{1}\right) I_{\lambda}$ possesses a global minimum

$\left(b_{2}\right)$ There is a sequence $\left\{u_{n}\right\}$ of critical points (local minimum) of $I_{\lambda}$ such that $\lim _{n \longrightarrow+\infty} \Phi\left(u_{n}\right)=+\infty$.

(c) If $\delta<+\infty$, then for each $\lambda \in(0,1 / \delta)$, the following alternative holds:

$\left(c_{1}\right)$ There is a global minimum of $\Phi$ which is a local minimum of $I_{\lambda}$

$\left(c_{2}\right)$ There is a sequence $\left\{u_{n}\right\}$ of pairwise distinct critical points (local minimum) of $I_{\lambda}$, with $\lim _{n \longrightarrow+\infty} \Phi\left(u_{n}\right)=\inf _{E} \Phi$, which weakly converges to a global minimum of $\Phi$.

Consider the $N$-dimensional Banach space:

$S=\left\{u:[0, N+1] \longrightarrow \mathbb{R}\right.$ such that $\left.u_{0}=u_{N+1}=0\right\}$,

endowed with the norm

$$
\|u\|=\left(\sum_{k=1}^{N+1}\left(\left|\Delta u_{k-1}\right|^{p}+2 q_{k}\left|u_{k}\right|^{p}\right)\right)^{1 / p} .
$$

We define another two norms on $S$ as follows:

$$
\begin{aligned}
\|u\|_{p} & =\left(\sum_{k=1}^{N+1}\left|\Delta u_{k-1}\right|^{p}\right)^{1 / p}, \\
\|u\|_{\infty} & =\max _{k \in \mathbb{Z}(1, N)}\left\{\left|u_{k}\right|\right\} .
\end{aligned}
$$

According to Lemma 2.2 in [12], we have the following inequality:

$$
\|u\|_{\infty}=\max _{k \in \mathbb{Z}(1, N)}\left\{\left|u_{k}\right|\right\} \leq \frac{(N+1)^{p-1 / p}}{2}\|u\|_{p}
$$

for every $u \in S$.

Lemma 2. For every $u \in S$, one has

$$
\|u\|_{\infty} \leq \frac{(N+1)^{p-1 / p}}{2(1+\underline{q})^{1 / p}}\|u\|
$$

where $\underline{q}=\min \left\{q_{k}, k \in \mathbb{Z}(1, N)\right\}$.

Proof. Making use of (11), we obtain 


$$
\begin{aligned}
\|u\|_{\infty}^{p}+\underline{q}\|u\|_{\infty}^{p} & \leq \frac{1}{2}\left(\left(\frac{N+1}{2}\right)^{p-1}\|u\|_{p}^{p}+\sum_{k=1}^{N+1} 2 q_{k}\left|u_{k}\right|^{p}\right) \\
& \leq \frac{(N+1)^{p-1}}{2^{p}}\|u\|^{p},
\end{aligned}
$$

then $\|u\|_{\infty} \leq(N+1)^{p-1 / p} / 2(1+\underline{q})^{1 / p}\|u\|$.

For every $u \in S$, put

$$
\begin{aligned}
& \Phi(u)=\frac{\|u\|^{p}}{p}, \\
& \Psi(u)=\sum_{k=1}^{N} F\left(k, u_{k+1}, u_{k}\right), I_{\lambda}(u)=\Phi(u)-\lambda \Psi(u),
\end{aligned}
$$

where $F(k, \cdot)$ satisfies the following conditions:

$\left(H_{2}\right) F(k, \cdot) \in C^{1}\left(\mathbb{R}^{2}, \mathbb{R}\right), F(k, 0,0)=0, F(0, . .)=$. $\forall k \in \mathbb{Z}(1, N)$

$\left(H_{3}\right) \partial F\left(k-1, x_{2}, x_{3}\right) / \partial x_{2}+\partial F\left(k, x_{1}, x_{2}\right) / \partial x_{2}=f(k$, $\left.x_{1}, x_{2}, x_{3}\right)$, for $\left(k, x_{1}, x_{2}, x_{3}\right) \in \mathbb{Z}(1, N) \times \mathbb{R}^{3}$

Direct computation ensures that $I_{\lambda}$ is a functional of class $C^{1}$ on $S$ with

$$
\frac{\partial I_{\lambda}}{\partial u_{k}}=-\Delta\left(\phi_{p}\left(\Delta u_{k-1}\right)\right)+2 q_{k} \phi_{p}\left(u_{k}\right)-\lambda f\left(k, u_{k+1}, u_{k}, u_{k-1}\right) \text {, }
$$$$
k \in \mathbb{Z}(1, N) .
$$

Then, $u$ is a critical point of $I_{\lambda}$ on $S$ if and only if

$$
-\Delta\left(\phi_{p}\left(\Delta u_{k-1}\right)\right)+2 q_{k} \phi_{p}\left(u_{k}\right)=\lambda f\left(k, u_{k+1}, u_{k}, u_{k-1}\right), \quad k \in \mathbb{Z}(1, N) .
$$

That is, the function $I_{\lambda}$ is just the variational framework of (1). For the reader's convenience, we recall a consequence of strong maximum principle [8]. The strong maximum principle is used to obtain positive solutions to (1), that is, $u_{k}>0$ for every $k \in \mathbb{Z}(1, N)$.

Lemma 3 (see [8]). Fix $u \in S$ such that

$$
\begin{array}{r}
\text { either } u_{k}>0 \text { or }-\Delta\left(\phi_{p}\left(\Delta u_{k-1}\right)\right)+q_{k} \phi_{p}\left(u_{k}\right) \geq 0, \\
\forall k \in \mathbb{Z}(1, N) .
\end{array}
$$

Then, either $u>0$ or $u \equiv 0$.

Remark 1. Assume $f\left(k, x_{1}, x_{2}, x_{3}\right): \mathbb{R}^{3} \longrightarrow \mathbb{R}$ is continuous for each $k \in \mathbb{Z}(1, N)$ and $\left(f\left(k, x_{1}, 0, x_{3}\right) \geq 0\right)$ for all $\left(k, x_{1}, x_{3}\right) \in \mathbb{Z}(1, N) \times \mathbb{R}^{2}$. Put

$$
f^{*}\left(k, x_{1}, x_{2}, x_{3}\right)= \begin{cases}f\left(k, x_{1}, 0, x_{3}\right), & \text { if } x_{2} \leq 0, \\ f\left(k, x_{1}, x_{2}, x_{3}\right), & \text { if } x_{2}>0,\end{cases}
$$

then $f^{*}(k, \cdot) \in C\left(\mathbb{R}^{3}, \mathbb{R}\right)$ for each $k \in \mathbb{Z}(1, N)$.

Consider the following boundary value problem:

$$
\left\{\begin{array}{l}
-\Delta\left(\phi_{p}\left(\Delta u_{k-1}\right)\right)+2 q_{k} \phi_{p}\left(u_{k}\right)=\lambda f^{*}\left(k, u_{k+1}, u_{k}, u_{k-1}\right), \\
\quad k \in \mathbb{Z}(1, N), \\
u_{0}=u_{N+1}=0 .
\end{array}\right.
$$

From Lemma 3, all solutions of (19) are either zero or positive; hence, they are also solutions for (1). When (19) possesses nontrivial solutions, (1) possesses positive solutions, independently of the sign of $f$.

\section{Main Results}

Put

$$
\begin{aligned}
Q & =\sum_{k=1}^{N} q_{k}, \\
\kappa & =\frac{1}{(1+Q)(N+1)^{p-1}}, \\
B & =\limsup _{\xi \rightarrow+\infty} \frac{\sum_{k=1}^{N-1} F(k, \xi, \xi)+F(N, 0, \xi)}{\xi^{p}} .
\end{aligned}
$$

Now, we consider the suitable oscillating behavior of $F\left(k, x_{1}, x_{2}\right)$ when $\left|x_{1}\right|^{p}+\left|x_{2}\right|^{p}$ goes to $+\infty$. We have the following theorem.

Theorem 1. Assume that $\left(\mathrm{H}_{2}\right)$ and $\left(\mathrm{H}_{3}\right)$ are satisfied and there exist two real sequences $\left\{a_{n}\right\}$ and $\left\{c_{n}\right\}$, with $\lim _{n \longrightarrow+\infty} c_{n}=+\infty$, such that

$$
\begin{aligned}
\left|a_{n}\right|^{p} & <\frac{2^{p-2}(1+\underline{q}) c_{n}^{p}}{(1+Q)(N+1)^{p-1}}, \quad \text { for all } n \in \mathbb{Z}, \\
A & =\liminf _{n \longrightarrow+\infty} \frac{\sum_{k=1}^{N} \max _{\left|x_{1}\right|^{p}+\left|x_{2}\right|^{p} \leq c_{n}^{p}} F\left(k, x_{1}, x_{2}\right)-\sum_{k=1}^{N-1} F\left(k, a_{n}, a_{n}\right)-F\left(N, 0, a_{n}\right)}{2^{p-2}(1+q) c_{n}^{p}-(1+Q)(N+1)^{p-1}\left|a_{n}\right|^{p}}<\kappa B .
\end{aligned}
$$

Then, problem (1) admits an unbounded sequence of solutions for each $\lambda \in\left((2+2 Q) / p B, 2 / p(N+1)^{p^{-1}} A\right)$.
Proof. Our aim is to apply Lemma $1(\mathrm{~b})$ to prove our conclusion. Fix $\lambda \in\left((2+2 Q) / p B, 2 / p(N+1)^{p-1} A\right)$, which 
clearly $\left(H_{1}\right)$ holds. Our conclusion needs to provide that $\gamma<+\infty$. Put $r_{n}=2^{p-1}(1+\underline{q}) c_{n}^{p} / p(N+1)^{p-1}$ for all $n \in Z$.
Owing to (12), if $\|u\| \leq\left(p r_{n}\right)^{1 / p}$, then $\left|u_{k+1}\right|^{p}+\left|u_{k}\right|^{p} \leq c_{n}^{p}$ for every $k \in \mathbb{Z}(1, N)$, and we obtain

$$
\begin{aligned}
\varphi\left(r_{n}\right) & =\inf _{\|u\| \leq\left(p r_{n}\right)^{1 / p}} \frac{\sup _{\|u\| \leq}\left(p r_{n}\right)^{1 / p} \sum_{k=1}^{N} F\left(k, u_{k+1}, u_{k}\right)-\sum_{k=1}^{N} F\left(k, u_{k+1}, u_{k}\right)}{r_{n}-\left(\|u\|^{p} / p\right)} \\
& \leq \inf _{\|u\| \leq\left(p r_{n}\right)^{1 / p}} \frac{\sum_{k=1}^{N} \max _{\left|u_{k+1}\right|^{p}+\left|u_{k}\right|^{p} \leq c_{n}^{p}} F\left(k, u_{k+1}, u_{k}\right)-\sum_{k=1}^{N} F\left(k, u_{k+1}, u_{k}\right)}{\left(2^{p-1}(1+q) c_{n}^{p} / p(N+1)^{p-1}\right)-\left(\|u\|^{p} / p\right)} .
\end{aligned}
$$

Let $\quad\left(g_{n}\right)_{k}=a_{n}$ for every $k \in \mathbb{Z}(1, N)$, and $\left\|g_{n}\right\|^{p}=2(1+Q)\left|a_{n}\right|^{p}$. From $(21)$, we obtain $\left\|g_{n}\right\| \leq\left(p r_{n}\right)^{1 / p}$, $\left(g_{n}\right)_{0}=\left(g_{n}\right)_{N+1}=0 . \quad$ Clearly, $\quad\left\{g_{n}\right\} \subset S \quad$ and $\quad$ and one has

$$
\begin{aligned}
& \varphi\left(r_{n}\right) \leq \frac{p(N+1)^{p-1}}{2} \frac{\sum_{\left|x_{1}\right|^{p}+\left|x_{2}\right|^{p} \leq c_{n}^{p}}^{N} F\left(k, x_{1}, x_{2}\right)-\sum_{k=1}^{N-1} F\left(k, a_{n}, a_{n}\right)-F\left(N, 0, a_{n}\right)}{2^{p-2}(1+\underline{q}) c_{n}^{p}-(1+Q)(N+1)^{p-1}\left|a_{n}\right|^{p}} . \\
& \quad I_{\lambda}\left(s_{n}\right)<\left(\frac{2+2 Q}{p}-\lambda(B-\varepsilon)\right) d_{n}^{p},
\end{aligned}
$$

Hence, $\gamma \leq \liminf _{n \rightarrow \infty} \varphi\left(r_{n}\right) \leq p(N+1)^{p-1} A / 2<+\infty$.

Now, we verify that $I_{\lambda}$ is unbounded from below. By (20), let $\left\{d_{n}\right\}$ be a positive real sequence with $\lim _{n \rightarrow \infty} d_{n}=$ $+\infty$ and

$$
B=\lim _{n \longrightarrow+\infty} \frac{\sum_{k=1}^{N-1} F\left(k, d_{n}, d_{n}\right)+F\left(N, 0, d_{n}\right)}{d_{n}^{p}} .
$$

First, we assume that $B=+\infty$, and then we can fix $M>(2+2 Q) / p \lambda$, and there exists $\nu_{M} \in \mathbb{Z}$ such that

$$
\sum_{k=1}^{N-1} F\left(k, d_{n}, d_{n}\right)+F\left(N, 0, d_{n}\right) \geq M d_{n}^{p}, \quad \forall n>v_{M} .
$$

We take a sequence $\left\{s_{n}\right\}$ in $S$ such that $\left(s_{n}\right)_{k}=d_{n}$ for every $k \in \mathbb{Z}(1, N),\left(s_{n}\right)_{0}=\left(s_{n}\right)_{N+1}=0$, and one has

$$
\begin{aligned}
I_{\lambda}\left(s_{n}\right) & =\Phi\left(s_{n}\right)-\lambda \Psi\left(s_{n}\right) \\
& =\frac{\left\|s_{n}\right\|^{p}}{p}-\lambda\left(\sum_{k=1}^{N-1} F\left(k, d_{n}, d_{n}\right)+F\left(N, 0, d_{n}\right)\right) \\
& <\left(\frac{2+2 Q}{p}-\lambda M\right) d_{n}^{p},
\end{aligned}
$$

for all $n>v_{M}$. That is, $\lim _{n \longrightarrow+\infty} I_{\lambda}\left(s_{n}\right)=-\infty$.

Next, we assume that $B<+\infty$. Since $\lambda>(2+2 Q) / p B$, from (25), we can fix $\varepsilon>0$ such that $\varepsilon<B-(2+2 Q) / p \lambda$, arguing as before, there is a $\nu_{\varepsilon} \in \mathbb{Z}$ such that

$$
\sum_{k=1}^{N-1} F\left(k, d_{n}, d_{n}\right)+F\left(N, 0, d_{n}\right) \geq(B-\varepsilon) d_{n}^{p}, \quad \forall n>v_{\varepsilon} .
$$

By choosing $\left\{s_{n}\right\}$ in $S$ as above, we obtain that for all $n>v_{\varepsilon}$. Hence, one has $\lim _{n \longrightarrow+\infty} I_{\lambda}\left(s_{n}\right)=-\infty$.

We have verified all assumptions of Lemma 1(b); then, there is a sequence $\left\{u_{n}\right\}$ of critical points (local minima) of $I_{\lambda}$ such that $\lim _{n \longrightarrow+\infty} \Phi\left(u_{n}\right)=+\infty$.

According to Theorem 1, it is easy to obtain the following corollary.

Corollary 1. Let $\left(\mathrm{H}_{2}\right)$ and $\left(\mathrm{H}_{3}\right)$ be satisfied, and assume that

$$
A=\liminf _{\xi \longrightarrow+\infty} \frac{\sum_{k=1}^{N} \max _{\left|x_{1}\right|^{p}+\left|x_{2}\right|^{p} \leq \xi^{p}} F\left(k, x_{1}, x_{2}\right)}{\xi^{p}}<2^{p-2}(1+\underline{q}) \kappa B .
$$

Then, problem (1) admits an unbounded sequence of solutions for each $\lambda \in\left((2+2 Q) / p B, 2^{p-1}(1+\underline{q}) /\right.$ $\left.p(N+1)^{p-1} A\right)$.

Proof. Let $\left\{c_{n}\right\}$ be a real sequence with $\lim _{n \longrightarrow \infty} c_{n}=+\infty$ such that

$$
A=\lim _{n \longrightarrow+\infty} \frac{\sum_{k=1}^{N} \max _{\left|x_{1}\right|^{p}+\left|x_{2}\right|^{p} \leq c_{n}^{p}} F\left(k, x_{1}, x_{2}\right)}{c_{n}^{p}} .
$$

We take $a_{n}=0$ for all $n \in \mathbb{Z}$ in (21); combining with $\left(H_{2}\right),(30)$ and $(31)$, we can apply Theorem 1 to reach the conclusion.

From the argument of Remark 1, we have the following theorem and corollary.

Theorem 2. If $f\left(k, x_{1}, 0, x_{3}\right) \geq 0$ for every $\left(k, x_{1}, x_{3}\right) \in$ $\mathbb{Z}(1, N) \times \mathbb{R}^{2}$ and the hypotheses of Theorem 1 hold. Then, problem (1) admits an unbounded sequence of positive solutions for each $\lambda \in\left((2+2 Q) / p B, 2 / p(N+1)^{p-1} A\right)$. 
Corollary 2. If $f\left(k, x_{1}, 0, x_{3}\right) \geq 0$ for every $\left(k, x_{1}, x_{3}\right) \in \mathbb{Z}(1, N) \times \mathbb{R}^{2}$ and the hypotheses of Corollary 1 are satisfied, then problem (1) admits an unbounded sequence of positive solutions for each $\lambda \in\left((2+2 Q) / p B, 2^{p-1}(1+\right.$ q)/p(N+1) $\left.)^{p-1} A\right)$.

Next, we consider the oscillating behavior of $F\left(k, x_{1}, x_{2}\right)$ when $\left|x_{1}\right|^{p}+\left|x_{2}\right|^{p}$ goes to 0 . We obtain the following theorem.

Theorem 3. Assume that $\left(\mathrm{H}_{2}\right)$ and $\left(\mathrm{H}_{3}\right)$ are satisfied and

$$
A^{*}<2^{p-2}(1+\underline{q}) \kappa B^{*}
$$

where $\quad A^{*}=\liminf _{\xi \longrightarrow 0^{+}}\left(\sum_{k=1}^{N} \max _{\left|x_{1}\right|^{p}+\left|x_{2}\right|^{p} \leq \xi^{p}} F\left(k, x_{1}\right.\right.$, $\left.\left.x_{2}\right)\right) / \xi^{p} \quad$ and $\quad B^{*}=\lim \sup _{\xi \longrightarrow 0+}\left(\sum_{k=1}^{N-1} F(k, \xi, \xi)+F(N\right.$,
$0, \xi)) / \xi^{p}$. Then, problem (1) possesses a sequence of pairwise distinct solutions, which converges to zero for each $\lambda \in\left((2+2 Q) / p B^{*}, 2^{p-1}(1+q) / p(N+1)^{p-1} A^{*}\right)$.

Proof. We will check the part (c) of Lemma 1. Fix $\lambda \in\left((2+2 Q) / p B^{*}, 2^{p-1}(1+q) / p(N+1)^{p-1} A^{*}\right)$, let $\left\{c_{n}\right\}$ be a positive real sequence such that $\lim _{n \longrightarrow \infty} c_{n}=0$ and

$$
A^{*}=\lim _{n \longrightarrow+\infty} \frac{\sum_{k=1}^{N} \max _{\left|x_{1}\right|^{p}+\left|x_{2}\right|^{p} \leq c_{n}^{p}} F\left(k, x_{1}, x_{2}\right)}{c_{n}^{p}} .
$$

As before, we let $r_{n}=2^{p-1}(1+q) c_{n}^{p} / p(N+1)^{p-1}$ for each $n \in Z$. In view of (12), note that $\|u\| \leq\left(p r_{n}\right)^{1 / p}$ implies $\left|u_{k+1}\right|^{p}+\left|u_{k}\right|^{p} \leq c_{n}^{p}$ for every $k \in \mathbb{Z}(1, N)$, by the definition of $\varphi$, we have

$$
\begin{aligned}
\varphi\left(r_{n}\right) & =\inf _{\|u\| \leq\left(p r_{n}\right)^{1 / p}} \frac{\sup _{\|u\| \leq}\left(p r_{n}\right)^{1 / p} \sum_{k=1}^{N} F\left(k, u_{k+1}, u_{k}\right)-\sum_{k=1}^{N} F\left(k, u_{k+1}, u_{k}\right)}{r_{n}-\|u\|^{p} / p} \\
& \leq \frac{p(N+1)^{p-1}}{2^{p-1}(1+q)} \frac{\sum_{k=1}^{N} \max _{\left|x_{1}\right|^{p}+\left|x_{2}\right|^{p} \leq c_{n}^{p}} F\left(k, x_{1}, x_{2}\right)}{c_{n}^{p}}
\end{aligned}
$$

then $\quad \delta \leq \liminf _{n \rightarrow \infty} \varphi\left(r_{n}\right) \leq p(N+1)^{p-1} A^{*} / 2^{p-1}(1+$ q) $<+\infty$.

Clearly, 0 is a global minimum of $\Phi$ in $S$, and $I_{\lambda}(0)=0$ by $\left(\mathrm{H}_{2}\right)$.

Moreover, we can verify that 0 is not a local minimum of $I_{\lambda}$. Given a positive real sequence $\left\{d_{n}\right\}$ with $\lim _{n \longrightarrow+\infty} d_{n}=0$ such that

$$
B^{*}=\lim _{n \longrightarrow+\infty} \frac{\sum_{k=1}^{N-1} F\left(k, d_{n}, d_{n}\right)+F\left(N, 0, d_{n}\right)}{d_{n}^{p}},
$$

if $B^{*}=+\infty$, fix $M>(2+2 Q) / p \lambda$, and there exists $v_{M} \in \mathbb{Z}$ such that

$$
\sum_{k=1}^{N-1} F\left(k, d_{n}, d_{n}\right)+F\left(N, 0, d_{n}\right)>M d_{n}^{p}, \quad \forall n>v_{M} .
$$

Let $\left\{v_{n}\right\} \subset S$ be a sequence satisfying $\left(v_{n}\right)_{k}=d_{n}$ for every $k \in \mathbb{Z}(1, N), \quad\left(v_{n}\right)_{0}=\left(v_{n}\right)_{N+1}=0$. Since $\left\|v_{n}\right\|=(2+2 Q)^{1 / p}$ $d_{n} \longrightarrow 0$ as $n \longrightarrow \infty$, we have

$$
\begin{aligned}
I_{\lambda}\left(v_{n}\right) & =\Phi\left(v_{n}\right)-\lambda \Psi\left(v_{n}\right) \\
& =\frac{\left\|v_{n}\right\|^{p}}{p}-\lambda\left(\sum_{k=1}^{N-1} F\left(k, d_{n}, d_{n}\right)+F\left(N, 0, d_{n}\right)\right) \\
& <\left(\frac{2+2 Q}{p}-\lambda M\right) d_{n}^{p}<0,
\end{aligned}
$$

for all $n>v_{M}$. Thus, 0 is not a local minimum of $I_{\lambda}$.
If $B^{*}<+\infty$, since $\lambda>(2+2 Q) / p B^{*}$, we can also find a positive real sequence $\left\{d_{n}\right\}$ with $\lim _{n \longrightarrow+\infty} d_{n}=0$ such that (35) holds. Fix $0<\varepsilon<B^{*}-(2+2 Q) / p \lambda$, and there exists $v_{\varepsilon} \in \mathbb{Z}$, such that

$$
\sum_{k=1}^{N-1} F\left(k, d_{n}, d_{n}\right)+F\left(N, 0, d_{n}\right)>\left(B^{*}-\varepsilon\right) d_{n}^{p}, \quad \forall n>v_{\varepsilon}
$$
that

Arguing as before, there exist a sequence $\left\{v_{n}\right\} \subset S$, such

$$
I_{\lambda}\left(v_{n}\right)<\left(\frac{2+2 Q}{p}-\lambda\left(B^{*}-\varepsilon\right)\right) d_{n}^{p}<0
$$

for all $n>v_{\varepsilon}$. Obviously, 0 is not a local minimum of $I_{\lambda}$, and $\left(c_{2}\right)$ of Lemma $1(c)$ is true.

A similar result to Theorem 2 is obtained as follows.

Theorem 4. If $f\left(k, x_{1}, 0, x_{3}\right) \geq 0$ for every $\left(k, x_{1}, x_{3}\right) \in \mathbb{Z}(1, N) \times \mathbb{R}^{2}$ and the hypotheses of Theorem 3 hold, then problem (1) admits a sequence of pairwise distinct positive solutions, which converges to zero for each $\lambda \in\left((2+2 Q) / p B^{*}, 2^{p-1}(1+q) / p(N+1)^{p-1} A^{*}\right)$.

\section{Examples}

Finally, we give two examples to illustrate our results.

Example 1. Consider the boundary value problem (1) with 


$$
\begin{aligned}
f\left(k, x_{1}, x_{2}, x_{3}\right)= & \left(1+\varepsilon+\cos \left(\varepsilon \ln \left(1+\left|x_{2}\right|^{p}+\left|x_{3}\right|^{p}\right)\right)\right. \\
& \left.-\varepsilon \sin \left(\varepsilon \ln \left(1+\left|x_{2}\right|^{p}+\left|x_{3}\right|^{p}\right)\right)\right) p\left|x_{2}\right|^{p-2} x_{2} \\
& +1+\varepsilon+\cos \left(\varepsilon \ln \left(1+\left|x_{1}\right|^{p}+\left|x_{2}\right|^{p}\right)\right) \\
& -\varepsilon \sin \left(\varepsilon \ln \left(1+\left|x_{1}\right|^{p}+\left|x_{2}\right|^{p}\right)\right) p\left|x_{2}\right|^{p-2} x_{2}
\end{aligned}
$$

$$
\begin{aligned}
F\left(k, x_{1}, x_{2}\right)= & \left(1+\left|x_{1}\right|^{p}+\left|x_{2}\right|^{p}\right)(1+\varepsilon+\cos \\
& \left.\cdot\left(\varepsilon \ln \left(1+\left|x_{1}\right|^{p}+\left|x_{2}\right|^{p}\right)\right)\right)-2-\varepsilon .
\end{aligned}
$$

Obviously, $F\left(k, x_{1}, x_{2}\right)$ satisfies conditions $\left(H_{2}\right)$ and $\left(H_{3}\right)$. It is easy to see that

$\max _{\left|x_{1}\right|^{p}+\left|x_{2}\right|^{p} \leq \xi^{p}} F\left(k, x_{1}, x_{2}\right)=\left(1+\xi^{p}\right)\left(1+\varepsilon+\cos \left(\varepsilon \ln \left(1+\xi^{p}\right)\right)\right)-2-\varepsilon$.

Clearly,

for $k \in \mathbb{Z}(1, N)$. Put

$$
\begin{aligned}
A & =\liminf _{\xi \longrightarrow+\infty} \frac{\sum_{k=1}^{N} \max _{\left|x_{1}\right|^{p}+\left|x_{2}\right|^{p} \leq \xi^{p}} F\left(k, x_{1}, x_{2}\right)}{\xi^{p}} \\
& =\liminf _{\xi \longrightarrow+\infty} \frac{\sum_{k=1}^{N}\left[\left(1+\xi^{p}\right)\left(1+\varepsilon+\cos \left(\varepsilon \ln \left(1+\xi^{p}\right)\right)\right)-2-\varepsilon\right]}{\xi^{p}} \\
& =N \liminf _{\xi \longrightarrow+\infty} \frac{\left(1+\xi^{p}\right)\left(1+\varepsilon+\cos \left(\varepsilon \ln \left(1+\xi^{p}\right)\right)\right)-2-\varepsilon}{\xi^{p}} \\
& =\varepsilon N,
\end{aligned}
$$

and

$$
\begin{aligned}
B= & \limsup _{\xi \longrightarrow+\infty} \frac{\sum_{k=1}^{N-1} F(k, \xi, \xi)+F(N, 0, \xi)}{\xi^{p}} \\
= & \limsup _{\xi \longrightarrow+\infty}\left[(2 N-1)(1+\varepsilon)+2(N-1) \cos \left(\varepsilon \ln \left(1+2 \xi^{p}\right)\right)\right. \\
& \left.+\cos \left(\varepsilon \ln \left(1+\xi^{p}\right)\right)\right] \\
\geq & (2 N-1) \varepsilon+2 \geq 2+\varepsilon .
\end{aligned}
$$

Let $\varepsilon$ be sufficiently small, then $(2+2 Q) / p B \leq(2+2 Q) /$ $(2+\varepsilon) p \leq 2^{p-1}(1+q) / p(N+1)^{p-1} \varepsilon N$. Hence, for each $\lambda \in\left((2+2 Q) / p B, 2^{\bar{p}^{-1}}(1+q) / p(N+1)^{p-1} \varepsilon N\right)$, (1) admits an unbounded sequence of solutions by Corollary 1 .
Moreover, we have $f\left(k, x_{1}, 0, x_{3}\right)=0$ for all $\left(k, x_{1}, x_{3}\right) \in \mathbb{Z}(1, N) \times \mathbb{R}^{2}$; according to Corollary 2, (1) admits an unbounded sequence of positive solutions.

Example 2. Consider the boundary value problem (1) with

$$
\begin{aligned}
f\left(k, x_{1}, x_{2}, x_{3}\right)= & {\left[\sin \left(\varepsilon \ln \left(\left|x_{2}\right|^{p}+\left|x_{3}\right|^{p}\right)\right)+\varepsilon \cos \right.} \\
& \cdot\left(\varepsilon \ln \left(\left|x_{2}\right|^{p}+\left|x_{3}\right|^{p}\right)\right)+\sin \\
& \cdot\left(\varepsilon \ln \left(\left|x_{1}\right|^{p}+\left|x_{2}\right|^{p}\right)\right)+\varepsilon \cos \\
& \left.\cdot\left(\varepsilon \ln \left(\left|x_{1}\right|^{p}+\left|x_{2}\right|^{p}\right)\right)+2+2 \varepsilon\right] \\
& p\left|x_{2}\right|^{p-2} x_{2},
\end{aligned}
$$

if $\left|x_{1}\right|^{2}+\left|x_{2}\right|^{2}+\left|x_{3}\right|^{2} \neq 0 \quad$ and $\quad f(k, 0,0,0)=0 \quad$ for $k \in \mathbb{Z}(1, N)$. Let

$$
F\left(k, x_{1}, x_{2}\right)= \begin{cases}\left(\left|x_{1}\right|^{p}+\left|x_{2}\right|^{p}\right)\left(\sin \left(\varepsilon \ln \left(\left|x_{1}\right|^{p}+\left|x_{2}\right|^{p}\right)\right)+1+\varepsilon\right), & \text { if }\left|x_{1}\right|^{2}+\left|x_{2}\right|^{2} \neq 0 \\ 0, & \text { if }\left|x_{1}\right|^{2}+\left|x_{2}\right|^{2}=0\end{cases}
$$


then $\left(H_{3}\right)$ holds. Clearly, $\left(H_{2}\right)$ holds and

$$
\max _{\left|x_{1}\right|^{p}+\left|x_{2}\right|^{p} \leq \xi^{p}} F\left(k, x_{1}, x_{2}\right)=\xi^{p}\left(\sin \left(\varepsilon \ln \xi^{p}\right)+1+\varepsilon\right) .
$$

By computation, we obtain

$$
\begin{aligned}
A^{*}= & \liminf _{\xi \longrightarrow 0^{+}} \frac{\sum_{k=1}^{N} \max _{\left|x_{1}\right|^{p}+\left|x_{2}\right|^{p} \leq \xi^{p}} F\left(k, x_{1}, x_{2}\right)}{\xi^{p}} \\
= & \liminf _{\xi \longrightarrow 0^{+}} \frac{\sum_{k=1}^{N} \xi^{p}\left(\sin \left(\varepsilon \ln \xi^{p}\right)+1+\varepsilon\right)}{\xi^{p}} \\
= & N \liminf _{\xi \longrightarrow 0^{+}}\left(\sin \left(\varepsilon \ln \xi^{p}\right)+1+\varepsilon\right) \\
= & \varepsilon N, \\
B^{*}= & \limsup _{\xi \longrightarrow 0^{+}} \frac{\sum_{k=1}^{N-1} F(k, \xi, \xi)+F(N, 0, \xi)}{\xi^{p}} \\
= & \limsup _{\xi \longrightarrow 0^{+}}\left[(2 N-1)(1+\varepsilon)+2(N-1) \sin \left(\varepsilon \ln 2 \xi^{p}\right)\right. \\
& \left.+\sin \left(\varepsilon \ln \xi^{p}\right)\right] \\
\geq & (2 N-1) \varepsilon+2 \geq 2+\varepsilon .
\end{aligned}
$$

Obviously, we have $(2+2 Q) / p B^{*} \leq(2+2 Q) /(2+\varepsilon) p \leq$ $2^{p-1}(1+q) / p(N+1)^{p-1} \varepsilon N$, when $\varepsilon$ be sufficiently small. On the contrary, $f\left(k, x_{1}, 0, x_{3}\right)=0$ for all $\left(k, x_{1}, x_{3}\right) \in$ $\mathbb{Z}(1, N) \times \mathbb{R}^{2}$.

The computations ensure that all the assumptions of Theorem 4 are satisfied. Then, for each $\lambda \in((2+2 Q) /$ $\left.p B^{*}, 2^{p-1}(1+q) / p(N+1)^{p-1} \varepsilon N\right),(1)$ admits a sequence of pairwise distinct positive solutions, which converges to zero.

\section{Data Availability}

No data were used to support this study.

\section{Conflicts of Interest}

The authors declare that there are no conflicts of interest regarding the publication of this paper.

\section{Authors' Contributions}

All authors contributed equally to the manuscript and read and approved the final manuscript.

\section{Acknowledgments}

This work was supported by the National Natural Science Foundation of China (Grant no. 11971126), Program for Changjiang Scholars and Innovative Research Team in University (Grant no. IRT_16R16), and Scientific and Technologial Innovation Programs of Higher Education Institutions in Shanxi (Grant no. 2019L0955).

\section{References}

[1] W. G. Kelly and A. C. Peterson, Difference Equations: an Introduction with Applications, Academic Press, Cambridge, MA, USA, 1991.

[2] R. P. Agarwal, Difference Equations and Inequalities: Theory, Methods, and Applications, Marcel Dekker, Inc., New York City, NY, USA, 2000.

[3] J. S. Yu and B. Zheng, "Modeling wolbachia infection in mosquito population via discrete dynamical models," Journal of Difference Equations and Applications, vol. 25, no. 11, pp. 1549-1567, 2019.

[4] G. Bonanno and P. Candito, "Nonlinear difference equations investigated via critical point methods," Nonlinear Analysis: Theory, Methods \& Applications, vol. 70, no. 9, pp. 3180-3186, 2009.

[5] G. Bonanno, P. Candito, and G. D'Aguì, "Variational methods on finite dimensional banach space and discrete problems," Advanced Nonlinear Studies, vol. 14, no. 4, pp. 915-939, 2014.

[6] G. Bonanno, P. Candito, and G. D’Aguì, "Positive solutions for a nonlinear parameter-depending algebraic system," Electronic Journal of Differential Equations, vol. 2015, no. 17, pp. 1-14, 2015.

[7] G. Bonanno, P. Jebelean, and C. Şerban, "Superlinear discrete problems," Applied Mathematics Letters, vol. 52, pp. 162-168, 2016.

[8] G. D’Aguì, J. Mawhin, and A. Sciammetta, "Positive solutions for a discrete two point nonlinear boundary value problem with p-laplacian," Journal of Mathematical Analysis and Applications, vol. 447, no. 1, pp. 383-397, 2017.

[9] R. P. Agarwal and R. Luca, "Positive solutions for a system of second order discrete boundary value problem," Advances in Difference Equations, vol. 470, p. 17, 2018.

[10] Z. Zhou and J. X. Ling, "Infinitely many positive solutions for a discrete two point nonlinear boundary value problem with фc-laplacian," Applied Mathematics Letters, vol. 91, pp. 28-34, 2019.

[11] J. X. Ling and Z. Zhou, "Positive solutions of the discrete dirichlet problem involving the mean curvature operator," Open Mathematics, vol. 17, no. 1, pp. 1055-1064, 2019.

[12] L. Q. Jiang and Z. Zhou, "Three solutions to dirichlet boundary value problems for $p$-laplacian difference equations," Advances in Difference Equations, vol. 2008, Article ID 345916, 10 pages, 2007.

[13] Z. Zhou and M. T. Su, "Boundary value problems for 2n-order $\phi c$-laplacian difference equations containing both advance and retardation," Applied Mathematics Letters, vol. 41, pp. 7-11, 2015.

[14] Y. H. Long and J. L. Chen, "Existence of multiple solutions to second-order discrete neumann boundary value problems," Applied Mathematics Letters, vol. 83, pp. 7-14, 2018.

[15] Y. H. Long and S. H. Wang, "Multiple solutions for nonlinear functional difference equations by the invariant sets of descending flow," Journal of Difference Equations and Applications, vol. 25, no. 12, pp. 1768-1789, 2019.

[16] S. H. Wang and Y. H. Long, "Multiple solutions of fourthorder functional difference equation with periodic boundary conditions," Applied Mathematics Letters, vol. 104, Article ID 106292, 2020.

[17] Z. M. Guo and J. S. Yu, "Existence of periodic and subharmonic solutions for second-order superlinear difference equations," Science in China Series A: Mathematics, vol. 46, no. 4, pp. 506-515, 2003. 
[18] Z. Zhou, J. S. Yu, and Z. M. Guo, "Periodic solutions of higher-dimensional discrete systems," Proceedings of the Royal Society of Edinburgh: Section A Mathematics, vol. 134, no. 5, pp. 1013-1022, 2004.

[19] P. Chen and H. Fang, "Existence of periodic and subharmonic solutions for second-order p-laplacian difference equations," Advances in Difference Equations, vol. 2007, Article ID 42530, 10 pages, 2007.

[20] Z. Zhou, J. S. Yu, and Y. M. Chen, "Periodic solutions of a 2nth-order nonlinear difference equation," Science in China Series A: Mathematics, vol. 53, no. 1, pp. 41-50, 2010.

[21] P. Mei and Z. Zhou, "Periodic and subharmonic solutions for a 2nth-order $p$-Laplacian difference equation containing both advances and retardations," Open Mathematics, vol. 16, no. 1, pp. 1435-1444, 2018.

[22] P. Mei, Z. Zhou, and G. H. Lin, "Periodic and subharmonic solutions for a $2 \mathrm{n}$ th-order $\varphi c$-laplacian difference equation containing both advances and retardations," Open Mathematics, vol. 12, no. 7, pp. 2085-2095, 2019.

[23] J. S. Yu, H. P. Shi, and Z. M. Guo, "Homoclinic orbits for nonlinear difference equations containing both advance and retardation," Journal of Mathematical Analysis and Applications, vol. 352, no. 2, pp. 799-806, 2009.

[24] P. Chen and X. H. Tang, "Existence of homoclinic orbits for 2nth-order nonlinear difference equations containing both many advances and retardations," Journal of Mathematical Analysis and Applications, vol. 381, no. 2, pp. 485-505, 2011.

[25] Z. Zhou and J. S. Yu, "Homoclinic solutions in periodic nonlinear difference equations with superlinear nonlinearity," Acta Mathematica Sinica, vol. 29, no. 9, pp. 1809-1822, 2013.

[26] G. H. Lin and Z. Zhou, "Homoclinic solutions in periodic difference equations with mixed nonlinearities," Mathematical Methods in the Applied Sciences, vol. 39, no. 2, pp. 245260, 2016.

[27] Q. Q. Zhang, "Homoclinic orbits for discrete hamiltonian Systems with indefnite linear part," Communications on Pure and Applied Analysis, vol. 14, no. 5, pp. 1929-1940, 2017.

[28] G. H. Lin and Z. Zhou, "Homoclinic solutions in non-periodic discrete $\phi$-laplacian equations with mixed nonlinearities," Applied Mathematics Letters, vol. 64, pp. 15-20, 2017.

[29] G. H. Lin and Z. Zhou, "Homoclinic solutions of discrete $\phi$-laplacian equations with mixed nonlinearities," Communications on Pure and Applied Analysis, vol. 17, no. 5, pp. 1723-1747, 2018.

[30] Q. Q. Zhang, "Homoclinic orbits for discrete hamiltonian systems with local super-quadratic conditions," Communications on Pure and Applied Analysis, vol. 18, no. 1, pp. 425-434, 2019.

[31] G. H. Lin, Z. Zhou, and J. S. Yu, "Ground state solutions of discrete asymptotically linear schrodinger equations with bounded and non-periodic potentials," Journal of Dynamics and Differential Equations, vol. 32, no. 2, pp. 527-555, 2020.

[32] D. Smets and M. Willem, "Solitary waves with prescribed speed on infinite lattices," Journal of Functional Analysis, vol. 149, no. 1, pp. 266-275, 1997.

[33] G. Bonanno and P. Candito, "Infinitely many solutions for a class of discrete nonlinear boundary value problem," Applicable Analysis, vol. 88, no. 4, pp. 605-616, 2009.

[34] G. Bonanno and B. G. Molica, "Infinitely many solutions for a boundary value problem with discontinuous nonlinearities," Boundary Value Problems, vol. 2009, Article ID 670675, 20 pages, 2009. 